\title{
Mapping the Macaque Superior Temporal Sulcus: Functional Delineation of Vergence and Version Eye-Movement-Related Activity
}

\author{
Matthew K. Ward, ${ }^{1,2}$ Mark S. Bolding, ${ }^{1,2}$ Kevin P. Schultz, ${ }^{1}$ and Paul D. Gamlin ${ }^{1,2}$ \\ ${ }^{1}$ Department of Vision Sciences and ${ }^{2}$ Center for the Development of Functional Imaging, University of Alabama at Birmingham, Birmingham, Alabama 35294
}

It is currently thought that the primate oculomotor system has evolved distinct but interrelated subsystems to generate different types of visually guided eye movements (e.g., saccades/smooth pursuit/vergence). Although progress has been made in elucidating the neural basis of these movement types, no study to date has investigated all three movement types on a large scale and within the same animals. Here, we used fMRI in rhesus macaque monkeys to map the superior temporal sulcus (STS) for BOLD modulation associated with visually guided eye movements. Further, we ascertained whether modulation in a given area was movement type specific and, if not, the modulation each movement type elicited relative to the others (i.e., dominance). Our results show that multiple areas within STS modulate during all movement types studied, including the middle temporal, medial superior temporal, fundus of the superior temporal, lower superior temporal, and dorsal posterior inferotemporal areas. Our results also reveal an area in dorsomedial STS that is modulated almost exclusively by vergence movements. In contrast, we found that ventrolateral STS is driven preferentially during versional movements. These results illuminate an STS network involved in processes associated with multiple eye movement types, illustrate unique patterns of modulation within said network as a function of movement type, and provide evidence for a vergence-specific area within dorsomedial STS. We conclude that producing categorically different eye movement types requires access to a common STS network and that individual network nodes are recruited differentially based upon the type of movement generated.

Key words: cortex; eye movements; fMRI; macaque; STS; vergence

\section{Introduction}

Due to the adaptive advantages conferred by the ability to monitor the environment dynamically and foveate targets of interest, it is thought that the primate oculomotor system has evolved distinct but interrelated subsystems to generate different types of volitional eye movements and that these saccadic, smooth pursuit, and vergence subsystems work in concert to direct gaze to, and maintain it on, objects of interest in 3D space (Gamlin, 2002; Krauzlis, 2004; Lynch and Tian, 2006). However, pure eye move-

Received Oct. 6, 2014; revised March 25, 2015; accepted March 31, 2015.

Author contributions: M.K.W. and P.D.G. designed research; M.K.W., M.S.B., and K.P.S. performed research; M.K.W. and M.S.B. contributed unpublished reagents/analytic tools; M.K.W. and K.P.S. analyzed data; M.K.W., M.S.B., K.P.S., and P.D.G. wrote the paper.

This work was supported by the W.M. Keck Foundation, NIH (Grants EB003292, NSF0116467, EY003039, EY018369, and EY022290), and the EyeSight Foundation of Alabama. We thank Sam Hayley and Jerry Millican for programming and material fabrication expertise, respectively; Debbie Whitten and Julie Hill for providing their expertise in animal care and procedures; Koen Nelissen for assistance; and Hoby Hetherington for providing the automated spherical harmonic shim routine and custom MP-RAGE sequence used in this study.

The authors declare no competing financial interests.

Correspondence should be addressed to Matthew K. Ward, Department of Ophthalmology, Shelby Building, 1170, 1825 University Blvd., University of Alabama at Birmingham, Birmingham, AL 35294. E-mail: mattward@uab.edu.

K. Schultz's and P. Gamlin's present affiliation: Department of Ophthalmology, University of Alabama at Birmingham, Birmingham, Alabama 35294.

M. Bolding's present affiliation: Department of Radiology, University of Alabama at Birmingham, Birmingham, Alabama 35294.

DOI:10.1523/JNEUROSCI.4203-14.2015

Copyright $\odot 2015$ the authors $\quad 0270-6474 / 15 / 357428-15 \$ 15.00 / 0$ ments of any type are rarely observed under natural viewing conditions. Therefore, to produce the combinatorial eye movements seen in naturalistic conditions, any subsystems related to those distinct movement types must be highly interconnected to rapidly integrate $3 \mathrm{D}$ position and motion information to produce appropriate eye movements in 3D space (for review, see Vanduffel et al., 2014).

Significant attention has been paid to determining how the nonhuman primate cerebral cortex controls eye movements in the frontoparallel plane (version), but the cortical network underlying eye movements to targets in depth (vergence) has received comparatively little attention, making a substantive comparison between their respective cortical networks limited at best. Several studies have described a cortical organization that is well suited to integrate movement-type-specific information originating from functionally distinct neural populations. Gamlin and Yoon (2000) redefined the extent and function of the rhesus macaque (Macaca mulatta) frontal eye field (FEF) by describing neurons located within the arcuate sulcus closely apposed to those involved in smooth pursuit and saccades, which were modulated by vergence movements and the sensorimotor transformations required for their production. Subsequently, neural subpopulations within caudal FEF (Fukushima et al., 2002), the supplementary eye field (Fukushima et al., 2004), and the middle superior temporal (MST) area (Akao et al., 2005) were shown to be modulated during either smooth pursuit alone, ver- 




Figure 1. Schematic of eye movement task conditions. Top left, Sinusoidal vergence tracking from $2^{\circ}$ verged to $8^{\circ}$ verged and back at $0.8 \mathrm{~Hz}$ for the duration of the block. Top right, Square-wave vergence steps from $2^{\circ}$ verged to $8^{\circ}$ verged and back at $0.8 \mathrm{~Hz}$ for the duration of the block. Bottom left, Sinusoidal smooth pursuit from $-6^{\circ}$ on the azimuth to $+6^{\circ}$ and back at $0.8 \mathrm{~Hz}$ for the duration of the block. Bottom right, Sequential saccades of 10 or $12^{\circ}$ at $1.5 \mathrm{~Hz}$ for the duration of the block.

gence tracking alone, or during both movement types. Importantly, these studies demonstrated how version and vergence movements might be generated from a common 3D representation of visuomotor space as opposed to three separate but cooperative systems. However, it is still undetermined whether this type of visuomotor mapping exists elsewhere in cortex.

Of the studies that have attempted to map cortex for visual sensitivity and/or control of volitional eye movements, the region of the macaque brain that has received much attention is the superior temporal sulcus (STS). Accordingly, we chose to target this region to identify areas related to several classes of visually guided eye movements and to determine how they fit into the mosaic of functional areas already mapped within the region by several different methods (Van Essen et al., 2001; Nelissen et al., 2006). The goals of this study were to delineate the spatial location and extent of eye-movement-related activity within the STS of the rhesus macaque monkey using BOLD-based fMRI, to determine whether eye-movement-related modulation therein could be attributed to a particular class of movement (vergence/ version) or, more specifically, to an individual movement type (vergence tracking/vergence steps/smooth pursuit/saccades) and to identify areas of interest.

\section{Materials and Methods}

Animals. Two male rhesus macaque monkeys (M1 and M2) were used in this study. All experiments were approved by the Institutional Animal Care and Use Committee of UAB and followed the National Institutes of Health's Guide for the Care and Use of Laboratory Animals.

Stimulus presentation and eye tracking. An Avotec dual display stimulus presentation system was used to deliver dichoptic visual stimuli to the animals and to track their eye position. Left and right eye position information was collected at $60 \mathrm{~Hz}$ (for offline analysis) using infrared cameras and pupil tracking software (ISCAN). At the start of every imaging session each animal's left and right eye positions and gain were calibrated with respect to targets moving $\pm 8^{\circ}$ along the vertical and horizontal axes. Accommodative demand was matched to a fixation target requiring the animal to be converged $2^{\circ}$.

Visual stimuli and experimental tasks. After implantation of an MRIcompatible head stabilization system (Gamlin et al., 2006), each animal was trained on 6 tasks, 2 requiring fixation only ( 1 including a simultaneously presented probe stimulus) and 4 requiring the animal to execute 
an eye movement in which they foveated a dichoptically presented white cross subtending $\sim 1^{\circ}$ of visual angle against a dark gray background (see Fig. 1 for a schematic of eye movement task types). All trial types began with the animal's eyes verged $2^{\circ}$ on the initial target and were presented continuously until the block ended or the animal's performance did not meet the task demands, at which point the next block began or the individual trial reset, respectively.

The first task required sustained fixation on a centrally located target (FIX) and served as the baseline for main effect comparisons. The second and third tasks required the animal to perform vergence eye movements to a target that moved from a position requiring $2^{\circ}$ of vergence to a position requiring $8^{\circ}$ of vergence (and back) at $0.8 \mathrm{~Hz}$ in either a sinusoidal (VERGENCE TRACKING) or stepwise (VERGENCE STEPS) fashion. The fourth task required the animal to perform smooth pursuit eye movements in response to a target moving in a sinusoidal fashion along the azimuth from $-6^{\circ}$ to $+6^{\circ}$ (and back) at $0.8 \mathrm{~Hz}$ (SMOOTH PURSUIT). The fifth task required the animal to perform serial saccades to targets presented at $1.5 \mathrm{~Hz}$ in the frontoparallel plane (SACCADE). SACCADE targets could appear at one of five different positions located at the vertices of a pentagon separated by $10^{\circ}$ of visual angle $\left(12^{\circ}\right.$ for nonadjacent targets). The sixth task required the animal to maintain fixation on a central target while a motion-in-depth probe was presented (a red cross subtending $\sim 1^{\circ}$ of visual angle and offset vertically to just above the fixation target). This probe stimulus moved in a sinusoidal fashion in depth from $1^{\circ}$ uncrossed disparity to $1^{\circ}$ crossed disparity (and back) at $0.8 \mathrm{~Hz}$ and was included to determine whether any modulation associated with the VERGENCE TRACKING task was related to the motion-in-depth component of the stimulus.

For all tasks, the animal was rewarded with a drop of juice for every 5-7 s of accurate eye position relative to target location. If the animal's eye position moved out of a $3^{\circ}$ target-centered window $\left(4^{\circ}\right.$ for SACCADE trials) for $>250 \mathrm{~ms}$, both the trial and the reward timer were reset. After the animals reached proficiency on all tasks $(\leq 2$ trial resets per $17 \mathrm{~s}$ epoch), they were acclimated in an aggregate fashion to the components of the scanner environment (i.e., head coil, visual display, ear protection, dark environment, body stabilization foam, and scanner noise; Gamlin et al., 2006). Once the animals were shaped to perform the experimental tasks proficiently in the presence of all scanner environment elements, they were considered ready for functional volume acquisition.

All functional runs followed an $\mathrm{ABC}$ block design (17 s block duration) with block A requiring only fixation and blocks $\mathrm{B}$ and $\mathrm{C}$ requiring either vergence and versional types, respectively (B: VERGENCE TRACKING or VERGENCE STEPS and C: SMOOTH PURSUIT or SACCADE) or tracking and stepped target trials, respectively (B: VERGENCE TRACKING or SMOOTH PURSUIT and C: VERGENCE STEPS or SACCADE). The scans involving the MIDprb condition were also conducted in an $\mathrm{ABC}$ block design with blocks $\mathrm{B}$ and $\mathrm{C}$ being comprised of the VERGENCE TRACKING task and the MIDprb task. To control for order effects, the composition of $\mathrm{B}$ and $\mathrm{C}$ blocks in all $\mathrm{ABC}$ functional runs were counterbalanced between runs.

Image acquisition. All experiments were conducted on a Varian $4.7 \mathrm{~T}$ vertical scanner running VnmrJ software. The monkey was seated in an upright, head-fixed position within a custom-made primate chair that supported left and right eye Avotec interface components. Each interface component contained a stimulus presentation screen, two embedded infrared illuminators, and an integrated infrared camera. Visual stimuli were conveyed into the magnet bore via coherent fiber optic bundles. All functional and anatomical volumes were acquired using a transmit/receive quadrature birdcage volume coil (Insight MRI). At the start of each imaging session, an automated spherical harmonic shim routine was used to both maximize homogeneity across the entire brain and to generate a B0 field map for offline correction of image warping (Miyasaka et al., 2006).

Monkeys were scanned using a one-shot (M1) or two-shot (M2) gradient-echo echoplanar imaging sequence with full brain coverage and a scan length of 100 volumes. A total of 7200 functional volumes were collected from each animal using the following acquisition parameters: TR: $2.1 \mathrm{~s}$; TE: $29 \mathrm{~ms} ; 64 \times 64$ matrix; resolution for M1: $2 \times 2 \times 2 \mathrm{~mm}$ voxels (33 axial-oblique slices), resolution for M2: $2 \times 2 \times 2.1$ voxels ( 30 axial-oblique slices). Four cycles of each $\mathrm{ABC}$ triad were completed within each successful functional run.

For each animal, a high-resolution anatomical brain volume was acquired to serve as that subject's common space for multirun/multisession analyses. Full-brain anatomical images were acquired using a custom MPRAGE sequence optimized for T1 contrast (TR: $2.5 \mathrm{~s}$; TI: $740 \mathrm{~ms}$; $256 \times 256$ matrix; $0.5 \times 0.5 \times 1 \mathrm{~mm}$ voxels; 70 axial slices). In addition, at the start of each imaging session, an anatomical volume with T1 contrast was collected using the same slice selection/voxel size parameters used for functional volume acquisition to serve as an intermediate point in the registration of functional volumes to high-resolution volumes. All functional data analyzed came from a total of 7000 functional volumes from M1, acquired in nine imaging sessions, and 7000 functional volumes from M2, acquired in six imaging sessions. Two runs from each animal's dataset were excluded from analysis due to excessive image artifacts.

Data analysis. All image preprocessing, GLM functional analyses, and volume-to-volume registrations (linear and nonlinear) were performed using tools found in the FMRIB Software Library (FSL) (Woolrich et al., 2001; Jenkinson et al., 2002; Smith, 2002; Jenkinson, 2003; Smith et al., 2004). Cortical surface generation and surface/slice overlays were performed using the Caret software package (Van Essen et al., 2001). Although full-brain functional volumes were initially collected, all analyses used a region of interest (ROI) to isolate and highlight the STS (except for the conjunction analysis results, which show full cortical maps to anchor the STS to other, well studied cortical oculomotor regions).

To remove nonbrain signal fluctuations, slow-wave fluctuations arising from scanner drift and high-frequency volume-to-volume fluctuations, functional time series were brain extracted and band-pass filtered before analysis. Functional time series were then tested for outlier volumes using the modified Thomson tau method with identified outlier volumes logged for later regression as variables of no interest. Brainextracted, band-pass-filtered functional time series were then motion corrected (MCFLIRT, rigid-body corrections) and unwarped (FUGUE, B0 map). Due to the relatively high CNR for functional runs and the need to maximize spatial specificity, no spatial smoothing was performed in the final analysis. There was no slice timing correction applied (although temporal derivatives were included in the GLM model) and all functional time series were prewhitened (FILM). Motion correction estimates, identified outlier volumes, and blocks containing three or more trial resets due to performance errors were included as covariates of no interest in the GLM model.

For each monkey, functional volumes underwent a two-stage linear registration process, first to the animal's daily anatomical volume via a rigid body transformation with three degrees of freedom and then to the animal's high-resolution volume via an affine transformation. Data from M2 underwent a final, nonlinear transformation into M1 brain space for group analyses. For surfaced-based images depicting group data, M1's anatomical volume was segmented using Caret's segmentation tool. From the resulting segmentation volume, cerebral hull, fiducial, inflated, and ellipsoid surfaces were generated. Finally, the ellipsoid surface was flattened using standard cuts for macaque monkey hemispheres.

Given the comparable task performance between animals (see Results), we investigated whether the data from the two animals could be pooled for group analysis based on the spatial extent and location of statistical maps. We conducted a conjunction analysis to identify voxels that were active for the same contrasts in both animals. For this and all subsequent group analyses, M2's brain was transformed into M1's brain space by first performing an affine registration of M2's anatomical volume to M1's anatomical volume (FMRIB's Linear Image Registration Tool, FLIRT). Subsequently, we performed a nonlinear registration of the resultant, affine-transformed anatomical volume of M2 back to M1's anatomical volume (FMRIB's Nonlinear Image Registration Tool, FNIRT). Finally, we applied the two transformation matrices resulting from the linear and nonlinear registrations to M2's functional volumes.

To generate the conjunction maps, all $\mathrm{EM}_{\text {ind }}>$ FIX contrasts from each animal (in common space) were thresholded at $p \geq 0.01_{\text {corr }}$ (voxelwise comparison in FSL) and subsequently binarized. The thresholded, binarized maps for each movement type were summed across animals and then 

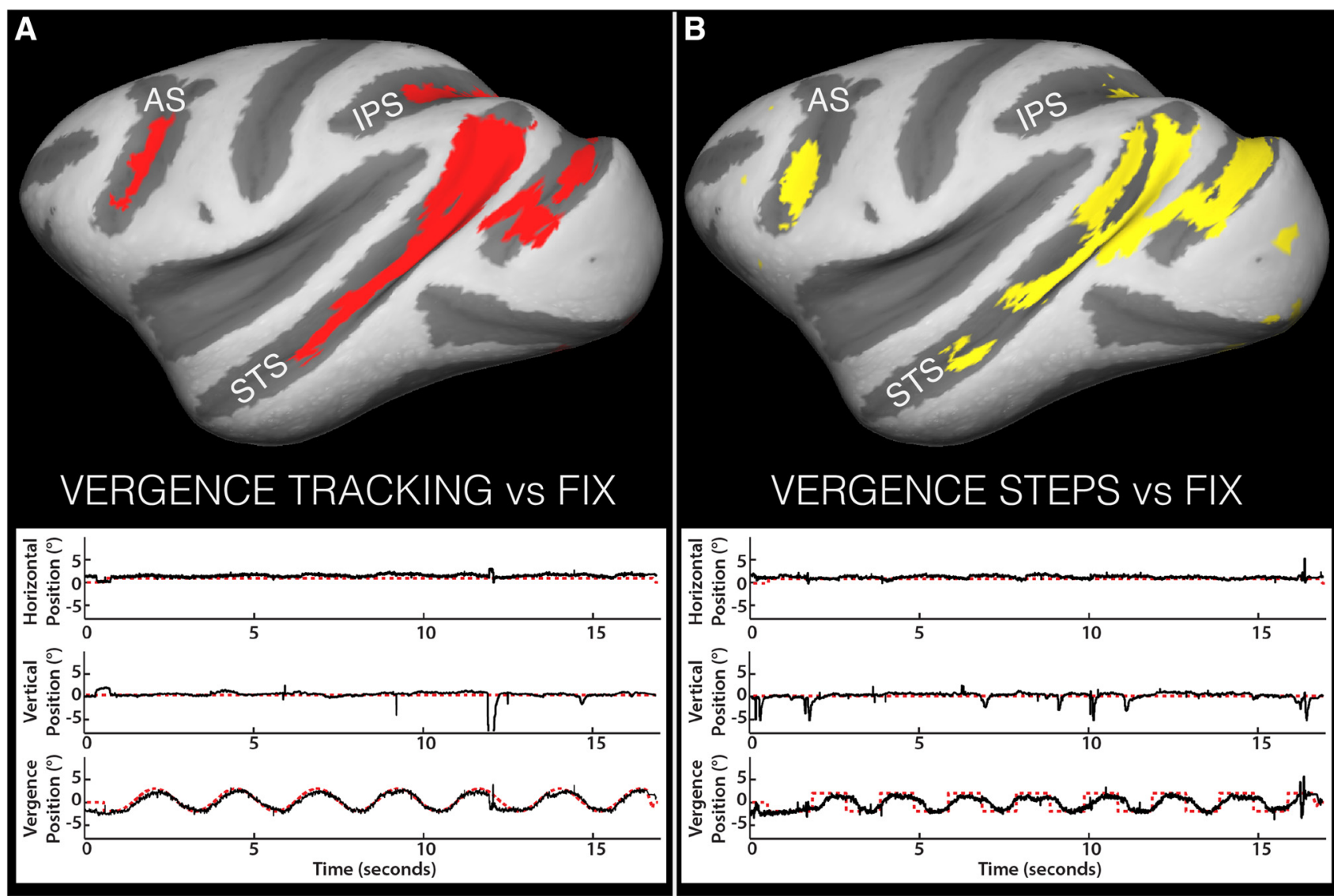

\section{VERGENCE STEPS vs FIX}
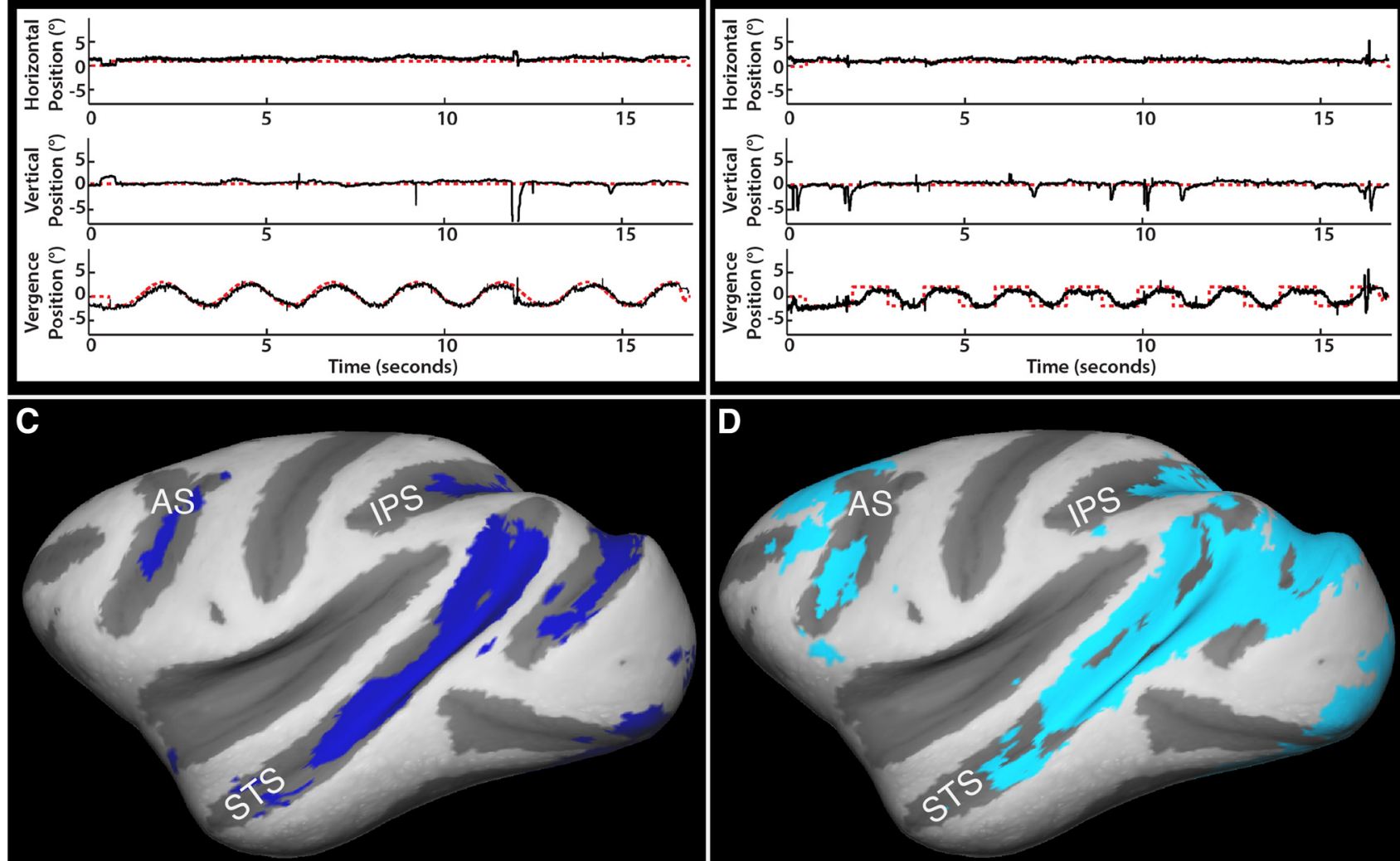

SMOOTH PURSUIT vs FIX

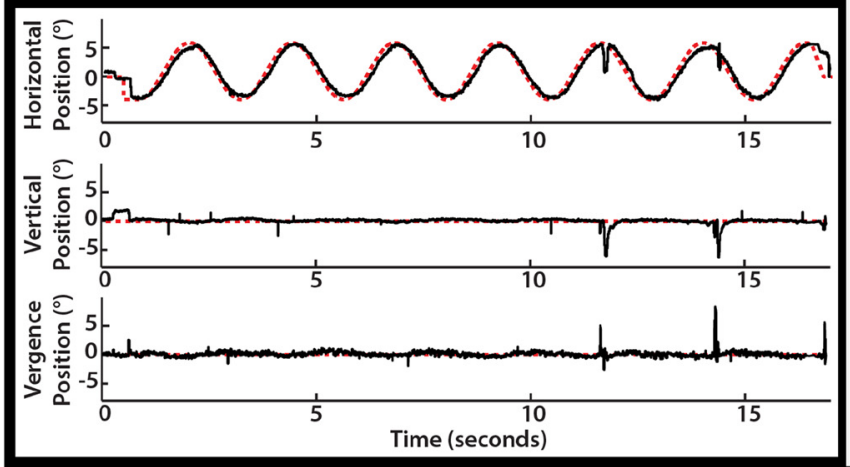

\section{SACCADES vs FIX}

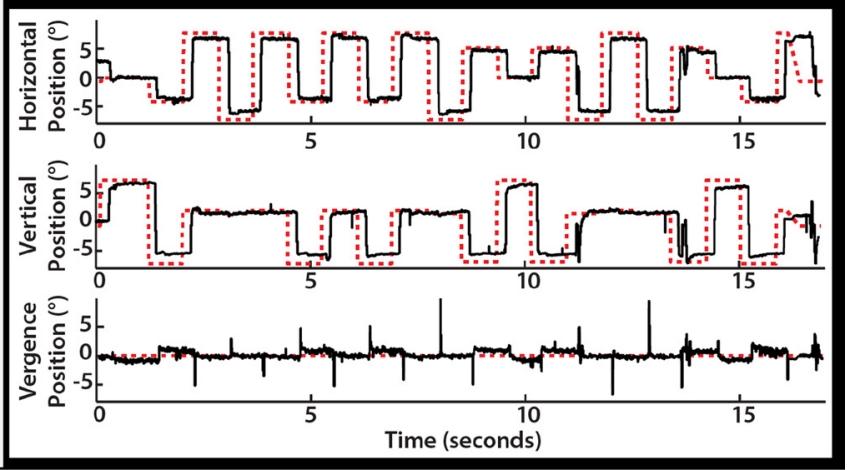

Figure 2. Eye-movement-specific conjunction maps overlaid on inflated cortical surfaces and representative eye/target position traces for each movement type. $A$, VERGENCE TRACKING $>$ Fix. $B$, VERGENCESTEPS > Fix. C, SMOOTH PURSUIT > Fix. D, SACCADE > Fix. Colored pixels represent voxels active for both animals for the given eye movement contrast. In the behavioral traces, target position is indicated by dashed red line, eye position by solid black line. AS, Arcuate sulcus; IPS, intraparietal sulcus. 
thresholded at 1.9, leaving only voxels active for a given contrast in both animals remaining. Figure 2 shows full cortical maps depicting the coarse location and extent of voxels active in both animals for each contrast. As can be seen, the familiar extrastriate areas that have previously been linked to visual and/or oculomotor behavior [e.g., STS, intraparietal sulcus (IPS), and periarcuate area] are identified in both animals for all eye movement types. To illustrate task performance (see Results), example eye position traces illustrating typical behavior are shown below each contrast map.

To further examine the degree of similarity of activity patterns between animals, we performed linear regression on the paired time points of each average time course for each eye movement type. Based on the equivalent task performance, the spatial congruence in their respective statistical maps and the high correlation coefficients resulting from the regressions (i.e., similar activation patterns between animals in a given area; see Results and Fig. $8 C$ ), data from the two monkeys were combined for group analyses.

Comparison types. Three comparison types were performed on the $4 \mathrm{D}$ time series acquired from the two animals. The purpose of the first comparison type was to identify all voxels within the STS in which activity was correlated to any, all, or some combination of eye movement types $\left(\mathrm{EM}_{\text {all }}\right.$ $>$ FIX, $F$ test). The purpose of the second comparison type was to partition eye-movement-related activity within the STS, as identified by EM $_{\text {all }}$ $>$ FIX, into eye movement-specific components by contrasting each eye movement type against fixation $\left(\mathrm{EM}_{\text {ind }}>\right.$ FIX, one-sample $t$ test $)$. In this manner, we could identify global similarities and gross differences in activity patterns as a function of eye movement type. The final comparison type contrasted each eye movement type against the other three types $\left(\mathrm{EM}_{\text {ind }}>\mathrm{EM}_{\text {ind }}\right.$; two-sample $t$ test). These contrasts were implemented to address any spatial overlap of signal modulation observed when comparing $\mathrm{EM}_{\text {ind }}>$ FIX maps and to establish eye-movementtype dominance in an area. These contrasts highlight spatial and modulation differences as a function of eye movement type while minimizing the representation of overlap. For one eye movement type, VERGENCE TRACKING, we wished to determine how much of the modulation in VERGENCE-TRACKING-related areas was due to the processing of disparity/MID signals. For this reason, we performed the contrast of VERGENCE TRACKING > MIDprb.

Contrasts comparing eye movement task conditions to fixation were thresholded at $p \geq 0.01_{\text {corr }}$ (voxelwise comparison in FSL) to maximize spatial specificity, whereas contrasts of eye movement task conditions against one another were thresholded at $p \geq 0.05_{\text {corr }}$ (voxelwise comparison in FSL) to minimize the occurrence of type 2 errors. Correction for multiple comparisons was performed using Gaussian random field theory-based maximum height thresholding.

Quantification of BOLD modulation within ROIs. Because statistical maps only tell part of the story, it is necessary to also look at the underlying signal dynamics to more fully understand what is occurring. Therefore, we investigated six statistically well defined ROIs within the STS [the posterior aspect of the ventral middle superior temporal area (MSTvp), the anterior aspect of the ventral middle superior temporal area (MSTva), dorsal middle superior temporal area (MSTd), middle temporal area (MT), fundus of the superior temporal (FST), and lower superior temporal (LST)] for the underlying BOLD signal changes associated with each eye movement type within each region. To maximize the amount of relevant information gained from the bilateral $3 \mathrm{~mm}$ ROI spheres, we used the eye movement contrast maps depicted in Figures $3 ; 4 ; 5 ; 6$, and 7 to inform their placement (see Fig. 8A). BOLD signal time courses were extracted from within the ROIs for each animal and normalized to baseline (i.e., fixation) to generate percent signal change values. From these individual time courses we generated average (across runs, across animals) BOLD signal time courses for each eye movement type within each of the six ROIs and sampled the middle four time points of each experimental task block to determine the maximum percent signal change. All experimental runs included in the GLM analyses were included in these quantification analyses.

Identification of eye-movement-related functional activation within the STS. To identify the clusters of eye-movement-related functional activations within the STS we transformed ROIs defined by motion sensitivity testing (msROIs; Nelissen et al., 2006) and applied them directly to our functional data. These msROIs included the designations of MSTd, MSTv, MT, FST, the middle portion of the superior temporal polysensory area (STPm), and LST. Given the deep, fundamental, and necessary relationship between the visual motion and oculomotor systems, embedding our eye movement results within motion-sensitive cortex provides a richer context in which to interpret their meaning.

To transform the msROIs from their native space into our common space, we conducted a two-stage registration process of our common space to that used in Nelissen's study. First, we performed an affine linear transformation of our anatomical volume to that from Nelissen's study. We then performed a nonlinear registration of the output from the linear registration to Nelissen's common space. Finally, we used the inverse of the transformation matrices (linear and nonlinear) to move the msROIs from their native space into our common space.

To corroborate identifications made using msROIs, to aid in the identification of functional activations that fell outside their borders, and, in some cases, to further subdivide functional activations into possible component parts, we transformed our functional data into the space of F99 found in the Caret software package (Van Essen, 2002) using the same method just described. This atlas provided functional parcellations based not only upon functional mapping studies, but also upon architectonic and connectivity data.

Finally, we used relative locations of nodes of activity and inspection of the visual cluster maps shown in Kolster et al. $(2009,2014)$ to further refine identification. Area labels associated with naming conventions other than that of Nelissen et al. are bounded by dashed lines in the figures.

\section{Results}

Our analysis of the BOLD signal within the STS during visually guided eye movements revealed that a large proportion of the sulcus, spanning much of the dorsomedial-to-ventrolateral extent, is modulated during one or more of the eye movement types tested. The borders of this expanse encompass multiple and distinct functional areas, some with previously described roles related to eye movements and some for which their role in the visuomotor system is still unclear.

\section{Task performance}

Both animals performed the experimental tasks as required. They fixated accurately during baseline blocks (within $1^{\circ}$ of target $\geq$ $95 \%$ of the time). For nonsaccade trial types, both the number of extraneous saccades (M1: $1.2 \pm 1.0, \mathrm{M} 2: 0.7 \pm 0.8)$ and blinks (M1: $2.9 \pm 1.3$, M2: $2.4 \pm 1.4$ ) per block were minimal. For saccade trial types, M1 and M2 completed an average of $19.7 \pm$ 1.7 and $19.5 \pm 1.9$ of a possible 21 saccades per block, respectively, with saccadic accuracy within $1^{\circ}$ of the target $>95 \%$ of the time. M1 and M2 performed vergence trial types accurately, remaining within $1^{\circ}$ of the target $\sim 95 \%$ of the time with gains of $0.95 \pm 0.07(\mathrm{M} 1)$ and $0.81 \pm 0.15(\mathrm{M} 2)$. For horizontal pursuit trials, M1's conjugate eye position remained within $1^{\circ}$ of the target $91 \pm 6 \%$ of the time with a gain of $1.05 \pm 0.02$, whereas $\mathrm{M} 2$ 's conjugate eye position remained within $1^{\circ}$ of the target $73 \pm$ $6 \%$ of the time with a gain of $0.97 \pm 0.02$.

\section{Eye-movement-responsive cortex within the macaque STS}

The results of our initial analysis determined the spatial extent within the STS that was responsive to visually guided eye movements of any type $\left(\mathrm{EM}_{\text {all }}>\right.$ FIX). The full extent of eyemovement-related modulation included areas MSTd, MSTv, MT, the transitional area of V4 (V4t), FST, LST and dorsal posterior inferotemporal (PITd). These results are informative in that they illustrate the occurrence of eye-movement-related BOLD signal modulation within a larger-than-expected proportion of the macaque STS. However, given the pooling of all eye 


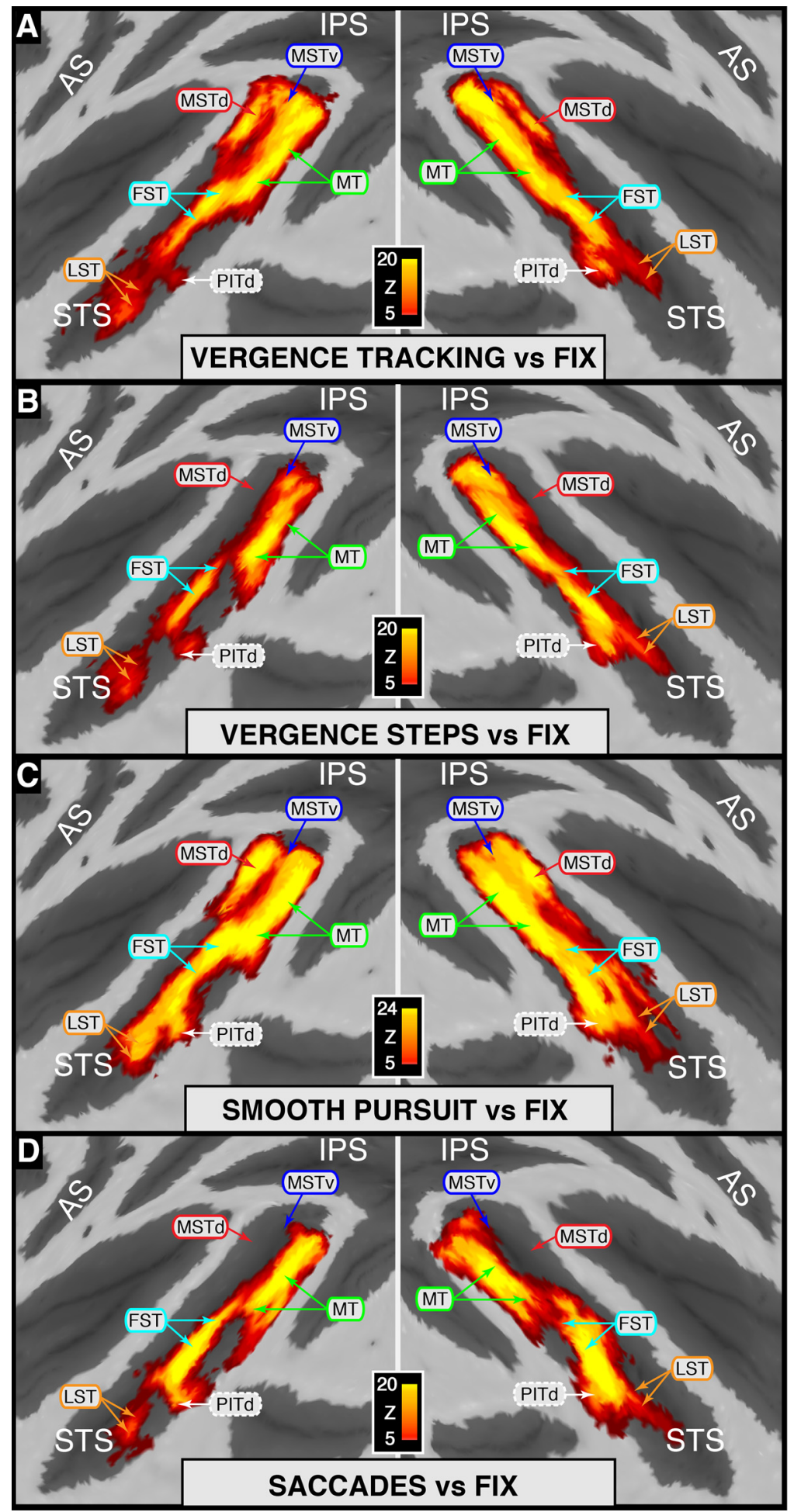

Figure 3. Statistical maps for individual eye movement types versus fixation overlaid on flattened cortical surfaces. $A$ VERGENCE TRACKING > Fix. B, VERGENCESTEPS > Fix.C, SMOOTH PURSUIT > Fix. D, SACCADE > Fix. Area identifiers shown with a solid border are based on Nelissen et al. (2006); area PITd is based on Kolster et al. $(2009,2014)$. For clarity, identifiers for V4t (bordering MT on the posterior bank/gyrus) were excluded from this figure and will be addressed in later figures. Because an STS ROI was used during GLM analyses, only activity within the STS is represented on these maps.

movement types and the wide range of statistical significance (i.e., $z$-scores), it cannot be determined from this contrast 1 ) which task/combination of tasks is causing the associated increase in local activity and 2), whether all distinct functional areas are represented, respectively. To address these issues, we per- formed contrasts consisting of each eye movement type alone compared with fixation $\left(\mathrm{EM}_{\text {ind }}>\right.$ FIX).

STS modulation by eye movement type Figure 3 depicts the full STS activation maps overlaid on flattened STS surfaces for each of the four eye movement types contrasted against fixation. Qualitatively, the STS maps for the four eye movement types look similar to one another in that many of the functional areas identified (MSTv, MT, FST, LST and PITd) show significant and bilateral modulation across all eye movement types tested. In contrast, gross differences can also be seen between eye movement types, such as the lack of MSTd activation for vergence steps and saccadic movements (Fig. $3 B, D$ ) and differences in statistical significance in some areas as a function of eye movement type (e.g., MT and MST modulation during SMOOTH PURSUIT carries greater statistical significance than does the modulation during VERGENCE TRACKING; note scale bars and color code). Visual inspection of the individual maps does not, however, give any insight into the relative strength of modulation in these areas with respect to specific eye movement types. To probe the robustness of modulation associated with each eye movement type in a given area relative to the other types, we performed direct contrast comparisons between each eye movement type and the other three types $\left(\mathrm{EM}_{\text {ind }}>\mathrm{EM}_{\text {ind }}\right)$.

\section{Preferential modulation by eye} movement type: vergence tracking Figure 4 shows cortical activation surface maps and selected coronal/sagittal slices highlighting where BOLD modulation is greater during VERGENCE TRACKING than for the other movement types. Figure $4 A$ depicts the results of contrasting VERGENCE TRACKING against SACCADE. This contrast revealed that areas MSTd, MSTv, and foveal MT are preferentially modulated during VERGENCE TRACKING compared with SACCADE. Within area MSTv, we found two peaks of activation, which we will tentatively refer to as posterior MSTv (MSTvp) and anterior MSTv (MSTva) based upon their positions relative to each other. MSTvp activation was located close to the fundus on the most dorsomedial portion of the anterior bank of the STS, whereas MSTva was located on the floor of the STS, $\sim 2 \mathrm{~mm}$ anterior to MSTvp (see sagittal slice ML -14 ; Fig. 4A).

Figure $4 B$ shows where VERGENCE TRACKING elicited more robust BOLD modulation than did SMOOTH PURSUIT. This contrast produced only one area, MSTvp, where BOLD sig- 


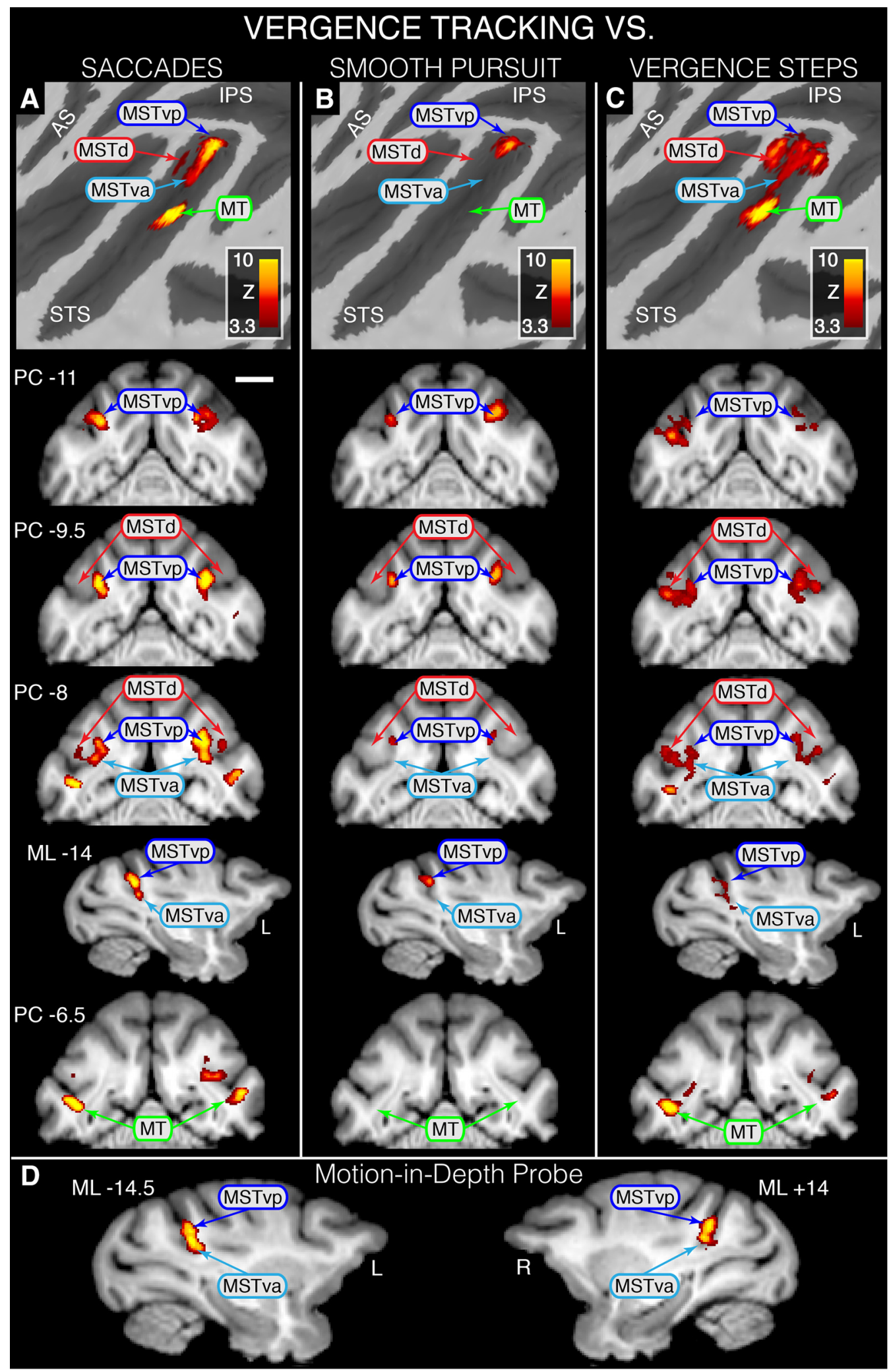

Figure 4. Statistical maps overlaid on flattened left cortical surfaces (top row) and selected slices showing where modulation during VERGENCE TRACKING is greater than that for the other eye movement types and MIDprb. A, VERGENCE TRACKING > SACCADE contrast revealing VERGENCE TRACKING dominance in MSTvp, MSTva and foveal MT. B, VERGENCE TRACKING > SMO0TH PURSUIT contrast revealing VERGENCE TRACKING dominance in MSTvp. C, D, VERGENCE TRACKING > VERGENCE STEPS contrast revealing VERGENCE TRACKING dominance in MSTd, MSTvp, MSTva, and foveal MT (C) and VERGENCE TRACKING > MIDprb revealing dominance in MSTd, MSTvd, and MSTva (D). All areas shown are based on Nelissen et al. (2006). For all coronal slices, position is relative to the posterior commissure (PC) with a negative value denoting a position caudal to PC; sagittal slice position is relative to the midline with a negative value denoting a position in the left hemisphere. Scale bar, $1 \mathrm{~cm}$. Because an STS ROI was used during GLM analyses, only activity within the STS is represented on these maps. 


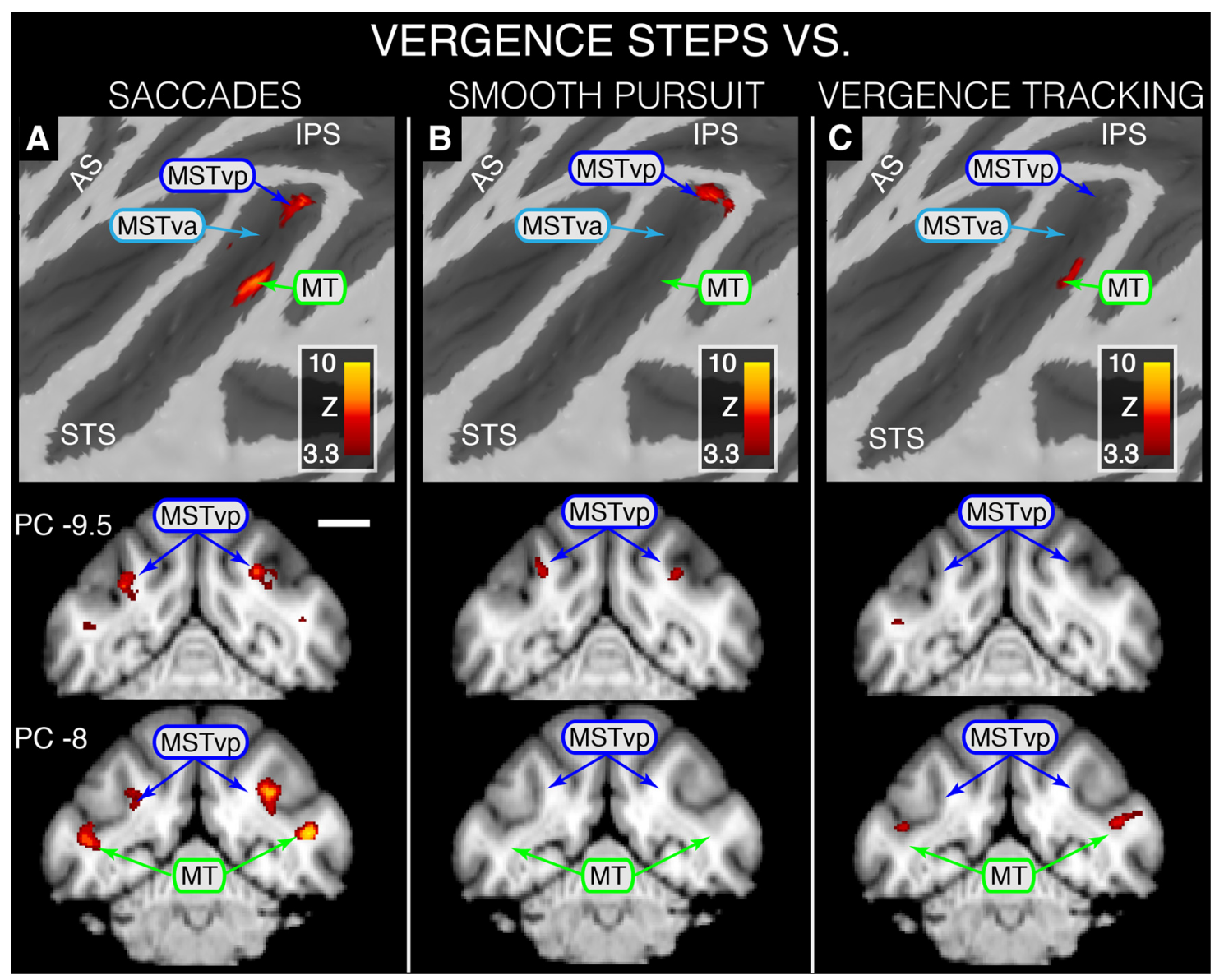

Figure 5. Statistical maps overlaid on flattened left cortical surfaces (top row) and selected slices showing where modulation during VERGENCE STEPS is greater than that for the other eye movement task conditions. $\boldsymbol{A}$, VERGENCE STEPS > SACCADE contrast revealing VERGENCE STEPS dominance in MSTvp, MSTva, and peripheral MT. B, C, VERGENCE STEPS > SMO0TH PURSUIT contrast revealing VERGENCE STEPS dominance in MSTvp $(\boldsymbol{B})$ and VERGENCE STEPS > VERGENCE TRACKING contrast revealing VERGENCE STEPS dominance in peripheral MT (C). All areas shown are based on Nelissen et al. (2006). Scale bar, $1 \mathrm{~cm}$. Because an STS ROI was used during GLM analyses, only activity within the STS is represented on these maps.

nal modulation was greater during the VERGENCE TRACKING task than for the SMOOTH PURSUIT task. This area overlaps substantially with the MSTvp area depicted in Figure 4A.

To determine whether the dynamics of the vergence movement (i.e., tracking vs stepped) caused differential modulation in any of the functional areas within the STS, we contrasted VERGENCE TRACKING against VERGENCE STEPS (Fig. $4 C)$. Indeed, this contrast resulted in an activation pattern similar to VERGENCE TRACKING > SACCADE, with greater modulation occurring in MSTd, MSTvp, MSTva, and foveal MT during VERGENCE TRACKING than during VERGENCE STEPS.

Finally, to determine whether the vergence-dominant activation within MSTvp and MSTva was influenced by the disparity processing associated with imperfect tracking of a target moving in depth, we compared VERGENCE TRACKING against MIDprb. The resulting map (Fig. 4D) shows that modulation due to VERGENCE TRACKING was greater than that for MIDprb within both MSTvp and MSTva.

\section{Preferential modulation by eye movement type: vergence} steps

Figure 5 depicts cortical surface activation maps and selected coronal slices highlighting where BOLD modulation is greater during VERGENCE STEPS than for the other three eye movement types. Figure $5 A$ shows that VERGENCE STEPS produces more robust modulation than SACCADE within MSTvp and MT, but, unlike the modulation associated with VERGENCE TRACKING (Fig. 4A), VERGENCE STEPS modulation within area MSTva is not significantly more robust than SACCADE.

The contrast of VERGENCE STEPS $>$ SMOOTH PURSUIT (Fig. 5B) illuminated only one area that was modulated more robustly during VERGENCE STEPS than for SMOOTH PURSUIT: area MSTvp. Compared with the results from the contrast of VERGENCE TRACKING $>$ SMOOTH PURSUIT, it can be seen that both VERGENCE STEPS and VERGENCE TRACKING produce more robust modulation in MSTvp than does SMOOTH PURSUIT, albeit to lesser degree for VERGENCE STEPS.

Finally; Figure $5 C$ shows that, when VERGENCE STEPS is contrasted against VERGENCE TRACKING, only a minimal area of MT shows more robust modulation.

\section{Preferential modulation by eye movement type: smooth} pursuit

Figure 6 summarizes the locations within the STS where BOLD signal modulation is greater for SMOOTH PURSUIT than for the other eye movement types. The results depicted in this figure indicate that, relative to the other eye movement types, the SMOOTH PURSUIT condition produced the most robust activation within the greatest number of functional areas along the length STS. Figure $6 A$ shows that, compared with VERGENCE 


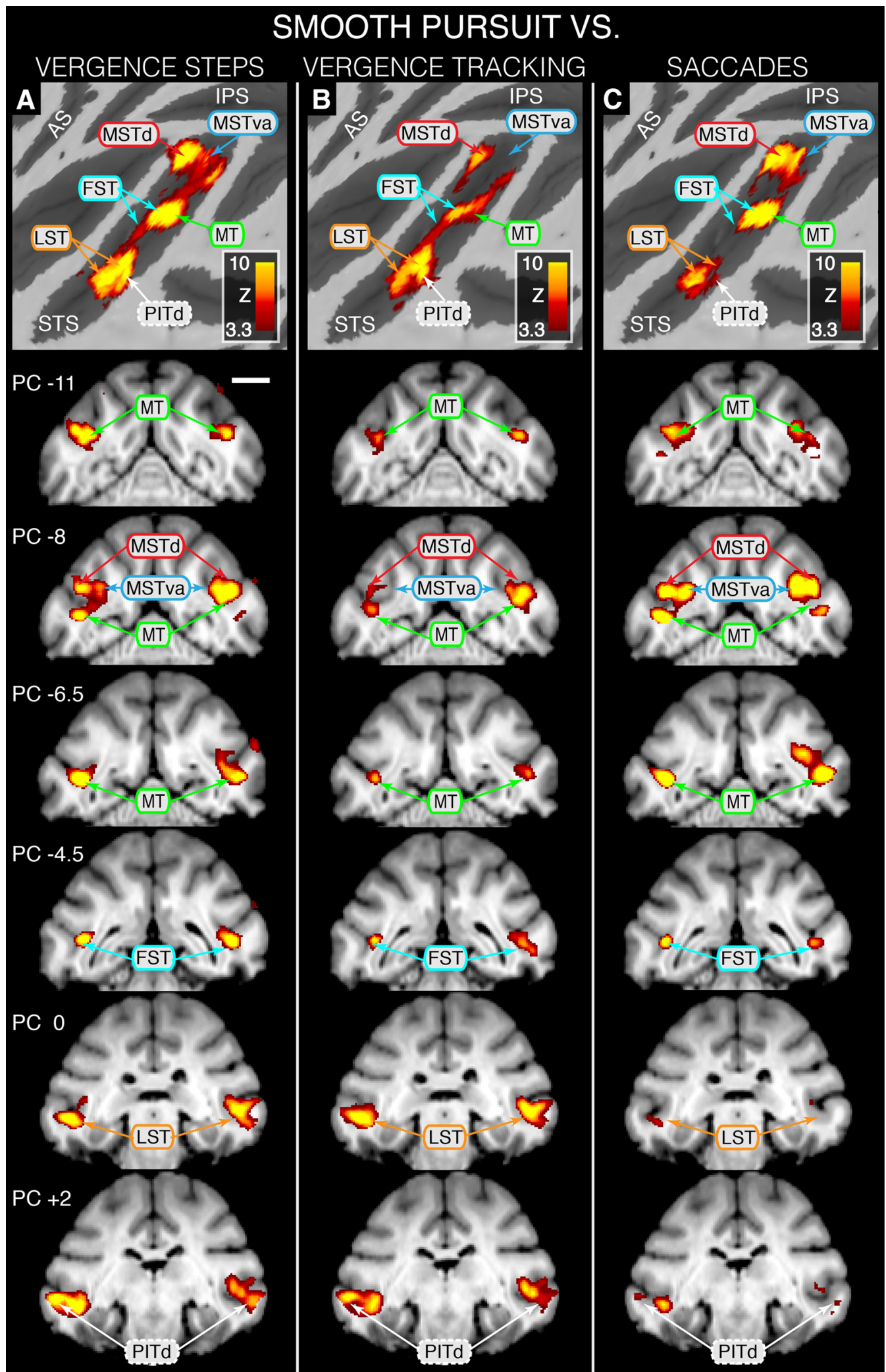

Figure 6. Statistical maps overlaid on flattened left cortical surfaces (top row) and selected slices showing where modulation during SMO0TH PURSUIT is greater than that for the other eye movement task conditions. $A$, SMOOTH PURSUIT > VERGENCE STEPS contrast revealing SMOOTH PURSUIT dominance in MSTdp, MSTd, MSTva, foveal and peripheral MT, FST, LST, and PITd. $B$, $C$, MOOTH PURSUIT > VERGENCE TRACKING contrast revealing SMOOTH PURSUIT dominance in MSTdp, MSTd, foveal and peripheral MT, FST, LST, and PITd (B) and SMO0TH PURSUIT > SACCADE contrast revealing SMOOTH PURSUIT dominance in MSTdp, MSTd, MSTva, foveal and peripheral MT, and FST (C). Area identifiers shown with a solid border are based on Nelissen et al. (2006); area PITd is based on Kolster et al. $(2009,2014)$. Scale bar. $1 \mathrm{~cm}$. Because an STS ROI was used during GLM analyses, only activity within the STS is represented on these maps. 


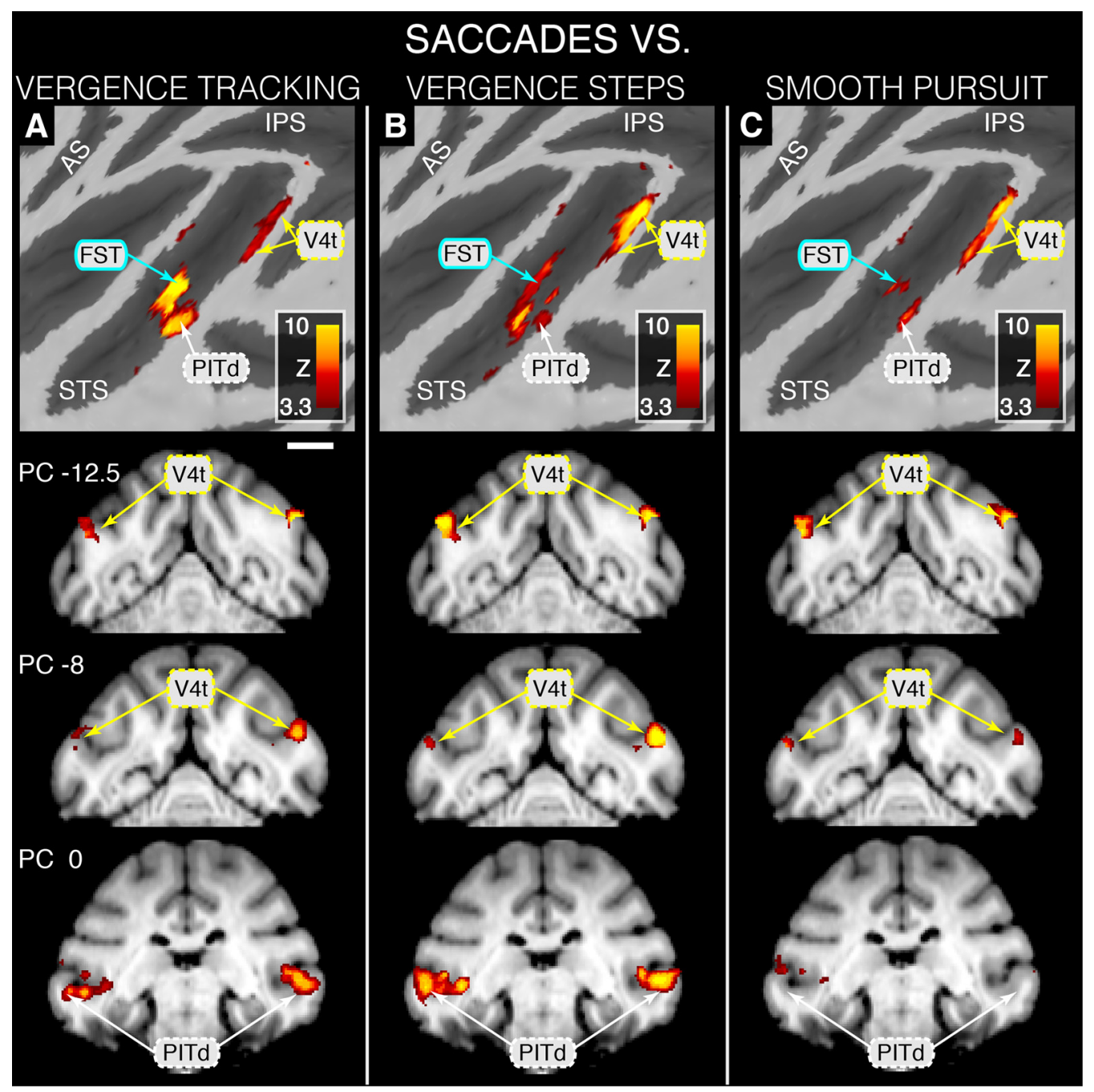

Figure 7. Statistical maps overlaid on flattened left cortical surfaces (top row) and selected slices showing where modulation during SACCADE is greater than that for the other eye movement task conditions. A, SACCADE > VERGENCE TRACKING contrast revealing SACCADE dominance in and PITd. B, SACCADE > VERGENCE STEPS contrast revealing SACCADE dominance in V4t and PITd. C, SACCADE > SMOOTH PURSUIT contrast revealing SACCADE dominance in V4t. Area FST is based on Nelissen et al. (2006), area V4t is based on Van Essen et al. (2001), and area PITd is based on Kolster et al. $(2009,2014)$. Scale bar, $1 \mathrm{~cm}$. Because an STS ROI was used during GLM analyses, only activity within the STS is represented on these maps.

STEPS, SMOOTH PURSUIT resulted in more robust BOLD modulation within the majority of identified areas (e.g., MSTd, MT, MSTva, FST, LST, and PITd).

The pattern of areas showing where SMOOTH PURSUIT induces greater BOLD modulation than VERGENCE STEPS (Fig. 6A) is largely preserved when contrasting SMOOTH PURSUIT against VERGENCE TRACKING except for two notable exceptions. The first is that MSTva does not show preferential modulation for SMOOTH PURSUIT when compared with VERGENCE TRACKING as it does when compared with VERGENCE STEPS. The second is that the statistical significances depicted in areas MSTd, MT, and FST (slices PC - 11 through PC -4.5 ) are smaller for the SMOOTH PURSUIT > VERGENCE TRACKING contrast than for the SMOOTH PURSUIT $>$ VERGENCE STEPS contrast, whereas being approximately equivalent between the two contrasts in the more ventrolateral areas of LST and PITd (slices PC 0 and PC +2).

Figure $6 \mathrm{C}$ highlights areas where BOLD signal modulation is more robust during SMOOTH PURSUIT than during SACCADE. As was also seen when contrasting SMOOTH PURSUIT against VERGENCE STEPS (Fig. 6A), the dorsomedial areas of MSTd, MSTva, and MT show clear preferential modulation during SMOOTH PURSUIT compared with SACCADE. In contrast to Figure $6 A$, however, there is minimal difference between modulation during the SMOOTH PURSUIT and SACCADE trial types in the ventrolateral regions of LST and PITd.

Preferential modulation by eye movement type: saccades

Figure 7 depicts BOLD-based activation patterns for the contrasts of SACCADE versus VERGENCE TRACKING, VERGENCE STEPS, and SMOOTH PURSUIT. There are relatively few areas that show differential and preferential modulation for SACCADE, but these areas persist across contrasts against the other movement types. For all three contrasts, SACCADE elicited greater modulation within area $\mathrm{V} 4 \mathrm{t}$ than did the other eye movement types (Figs. $7 A-C$; slices PC -12.5 and PC -8 ). Areas FST, LST, and PITd displayed preferential modulation during SACCADE blocks when contrasted against VERGENCE TRACKING (Fig. 7A) and VERGENCE STEPS (Fig. $7 B$ ), but showed weak and unilateral dominance in these areas when contrasted against SMOOTH PURSUIT (Fig. 7C).

Overview of eye movement to eye movement contrasts: areas of special interest

The overall findings from eye movement against eye movement contrasts suggest that, compared with versional eye movements, 
disparity-driven vergence eye movements more robustly modulate the BOLD signal in the most dorsomedial aspect of the STS along the fundus and anterior bank (MSTvp; Figs. 4, 5). Conversely, versional eye movements produce greater modulation in the ventrolateral aspect of the sulcus (LST, PITd; Figs. 6, 7). With respect to tracking movements and the caudal STS, area MSTvp was most robustly modulated during vergence tracking, MSTva was equally modulated during both frontal (smooth pursuit) and in-depth pursuit (vergence tracking), whereas area MSTd was preferentially modulated during SMOOTH PURSUIT. When considering the entirety of the STS, smooth pursuit movements elicited significant signal modulation within all areas identified, with the modulation being greater than that elicited by other movements for most of the areas identified (Fig. 6). The results pertaining to smooth pursuit movements suggest that the SMOOTH PURSUIT condition could provide an efficient functional localizer for multiple visual areas within the STS.

\section{BOLD signal modulation profiles and subject-to-subject correlation}

To characterize the BOLD signal modulations underlying the statistical maps we have shown here and to compare the modulations observed between the two individual animals, we extracted BOLD signal time courses from six ROIs from each animal: MSTvp, MSTva, MSTd, MT, FST, and LST. Figure $8 A$ depicts the locations of the ROIs used for signal extraction (larger green circles result from slice going through the middle of the sphere; smaller green circles result from slice going through the periphery of the sphere; all ROIs are bilateral). Figure $8 B$ shows the BOLD signal percent signal change for each movement type in each ROI averaged across all runs from both animals. Although the individual areas displayed differential activation patterns with respect to the different eye movement types, each area was modulated to some degree during all of the eye movement tasks (although a few did not reach our statistical threshold, i.e., VERGENCE STEPS and SACCADES within MSTd). Generally speaking, pursuit movements produced the greatest degree of modulation within the six ROIs except for the most dorsomedial (i.e., MSTvp) and ventrolateral (i.e., LST) ROIs, where there appears to be a preference for vergence and version movements, respectively, rather than for pursuit over stepped movements as is seen in MSTva, MSTd, MT, and FST. Figure $8 C$ illustrates the similarity in response patterns between $\mathrm{M} 1$ and $\mathrm{M} 2$ in each area for each movement type.

\section{Discussion}

Visually guided eye-movement-related cortex within the STS As Figures 2 and 3 show, a large number of areas within macaque STS exhibited MR signal modulation in response to each of the four eye movement types tested. Each of these areas has been previously associated with the visuomotor system by one or more of several criteria (e.g., functional, connectivity; Dürsteler and Wurtz, 1988; Komatsu and Wurtz, 1988a, 1988b, 1989; Stanton et al., 2005; Bakola et al., 2007; Distler and Hoffman, 2008; Hoffmann et al., 2009). However, related studies generally used only one type/class of eye movement, requiring cross-study comparisons of different movement types (along with the inherent difficulties of such comparisons) to gain insight into the movements' respective networks. Here, we demonstrated that, despite using the same simple visual stimulus to guide eye movements and despite the simple target motion profiles used, activity patterns indicate that many functional areas within STS are recruited dur- ing all eye movements studied, albeit to varying degrees and with some interesting exceptions.

Figure $8 B$ shows that, although the majority of areas revealed in the spatial maps were significantly modulated during all movement types, it also shows that areas within STS can be differentiated based upon whether an eye movement occurs in depth (i.e., MSTvp), in the frontoparallel plane (i.e., LST), and/or contains a smooth tracking component (i.e., MSTva). Together, these observations support the conclusion by Krauzlis (2004) in reference to saccadic and smooth pursuit movements that, instead of viewing the two movement types, “... as two distinct neural systems, it may be more accurate to consider the two movements as different outcomes from a shared cascade of sensory-motor functions." Further, much as Gamlin and Yoon (2000) expanded the executive reach of the FEF to include vergence movements, here, we find in early extrastriate cortex that areas involved in vergence eye movements also have roles in producing saccadic and smooth pursuit movements.

\section{MST-pursuit movements in 3D space}

This study provides evidence that area MST, known for its subdivisions based upon motion sensitivities, can also be subdivided based upon the visual plane in which an eye movement occurs (in-depth or in the frontoparallel plane) and whether it is a stepped or pursuit movement. Given that a difference between our vergence and version pursuit types was changing target disparity during vergence, one possible interpretation is that processing changing disparity underlies the vergence dominance in MSTvp. This is unlikely because the vergence dominance easily persisted when contrasting vergence tracking against a changing disparity-based motion-in-depth probe (Fig. 4D). This finding suggests the presence of extraretinal signals in this area associated with vergence movements, such as were found in MSTd for smooth pursuit (Newsome et al., 1988). To understand the significance of this vergence-dominant area in the context of the classical motion complex, it is necessary to examine the pattern of activity within area MSTva.

Compared with MSTvp, activity within MSTva was closely associated with pursuit movements because it was modulated strongly during both vergence tracking and smooth pursuit when compared with fixation but modulated equally when compared with each other (Figs. 4, 6). These results indicate that, much like areas FEF and SEF, area MSTva may contain cell populations that play a role in either tracking in depth, tracking within the frontoparallel plane, or both, an ideal configuration for keeping moving targets in 3D space foveated. The classification of this area as pursuit related is supported by work in MSTv by Tanaka et al. (1993) and in MSTl by Eifuku and Wurtz $(1998,1999)$. Tanaka et al. (1993) concluded that MSTv neurons were involved in the processing object motion in external space. Building upon Tanaka's results, Eifuku and Wurtz $(1998,1999)$ described a receptive field organization of cells in this area (MSTl) that indicate a role in the processing of object motion and segmenting moving objects from the background, critical properties for accurate pursuit generation. In support of this conclusion (at least for vergence tracking but possibly also for smooth pursuit), this area is modulated more during the movement than for the stimulus alone.

Area MSTd showed the most robust modulation during tracking movements compared with stepped movements (Figs. 3, 6), with the greatest modulation occurring during smooth pursuit. It is well established that area MSTd is essential for decoding optic flow (Duffy and Wurtz, 1991) and that such a role is of special 

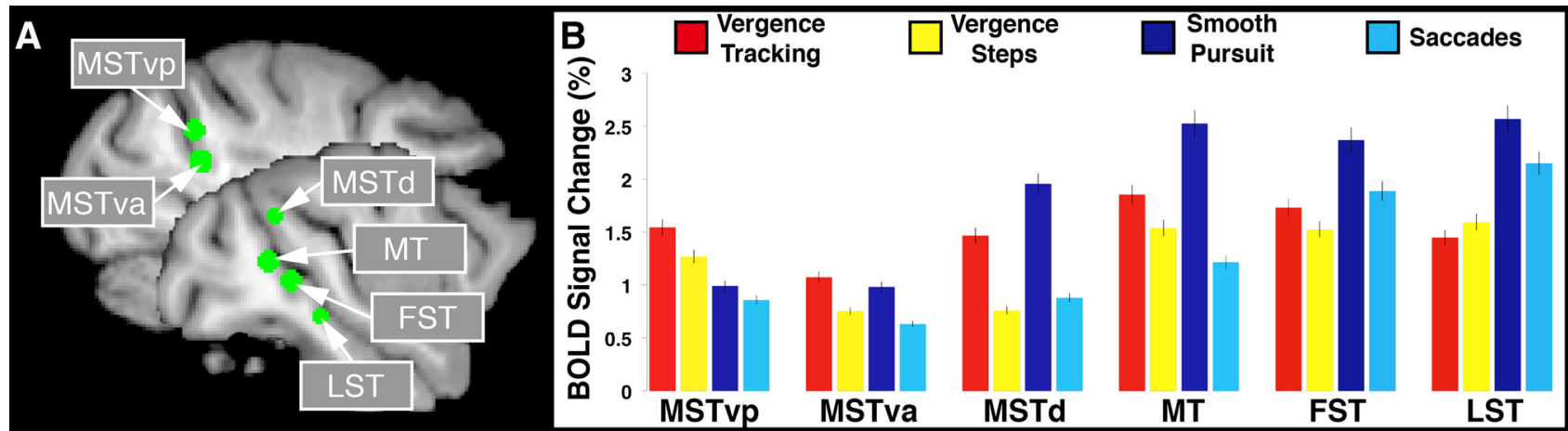


Vergence Steps
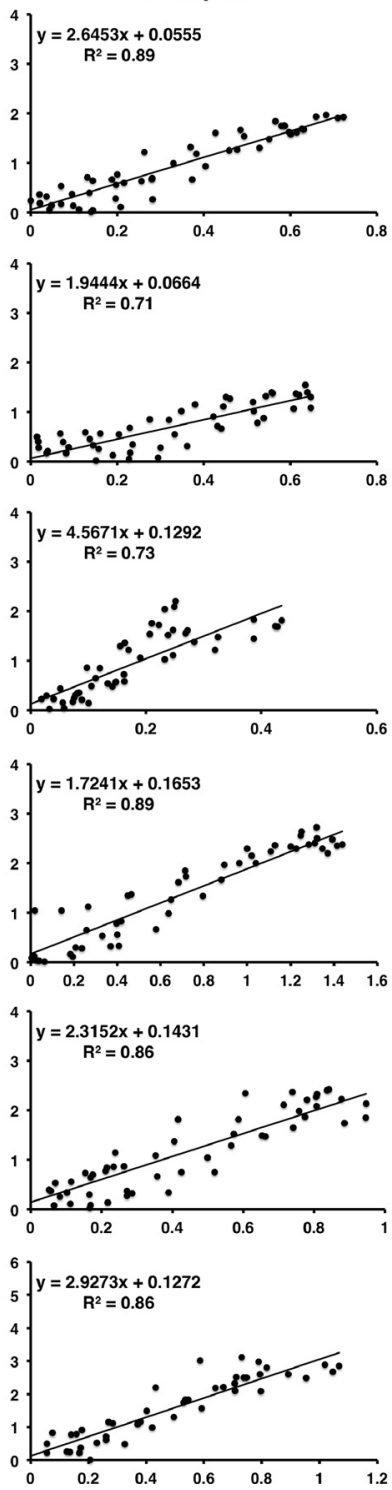

\section{Smooth \\ Pursuit}


\section{Saccades}
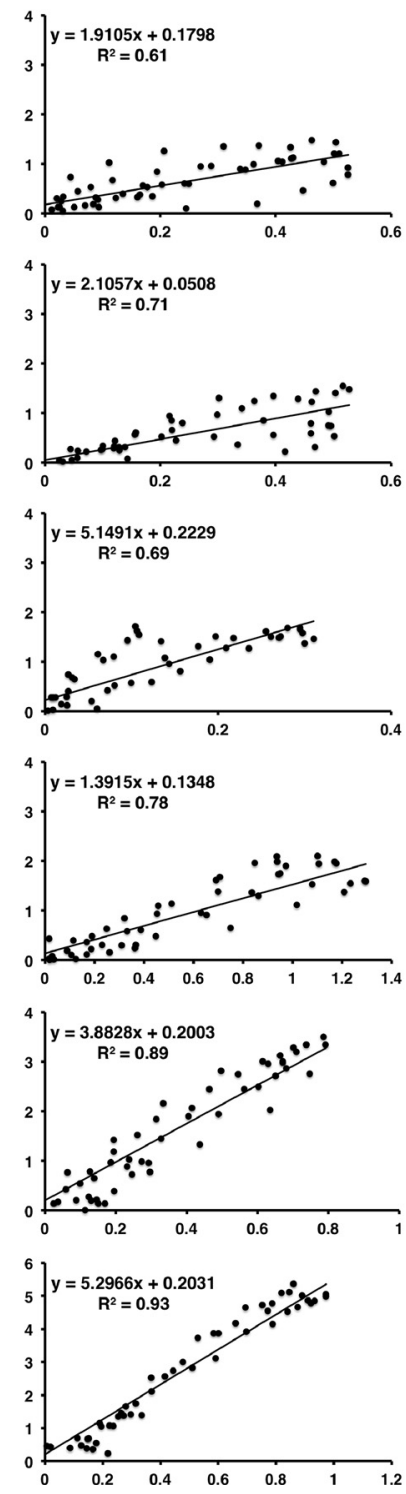

Figure 8. ROl locations, BOLD signal modulation profiles, and measures of intersubject agreement. $A$, Location of the ROls used for BOLD signal extraction (green circles) overlaid on left hemisphere sagittal slices. $\boldsymbol{B}$, Percent signal change values for all eye movement types within all six ROls. $\boldsymbol{C}$, Correlation between the animals' averaged time courses for each eye movement type in each region. The $x$-axis represents M1's data and the $y$-axis represents M2's data in all regression plots.

relevance for differentiating self-induced and externally induced retinal image motion (Zemel and Sejnowski, 1998). Although MSTd was preferentially activated during tracking movements compared with stepped movements, SMOOTH-PURSUIT- related modulation was greater than that for VERGENCE TRACKING. These results indicate that MSTd is modulated most efficiently during frontoparallel pursuit and provides another metric by which to differentiate and identify this area. 
The overall modulation pattern seen in MST suggests that there may be a visuomotor mapping for tracking movements in 3D space within dorsomedial STS, a cortical framework posited previously by Fukushima et al. $(2002,2004)$ and Akao et al. (2005). Further, Breveglieri et al. (2012) demonstrated the presence of cells within area V6a responsive to vergence and version and hypothesized that they encoded gaze positions in $3 \mathrm{D}$ space. Recently, this framework has been extended to the subcortical domain by Van Horn et al. (2013), who described neurons in the rostral superior colliculus that code for smooth eye movements in $3 \mathrm{D}$ space. However, although there may be a $3 \mathrm{D}$ visuomotor map organizing pursuit movements within caudal STS, based upon the retinotopy work in STS by Kolster et al. (2009, 2014) and their demonstration of visual field map clusters, the components of a 3D pursuit visuomotor map would fall into at least two different visual map clusters as MSTvp and MSTd fall outside of the MT cluster. Interestingly, Kolster et al. describe two new hemifield representations adjacent to but separate from the MT cluster (one ventral to V4t and LST, the other located dorsal to MT and MSTv). Within the context of our results, MSTvp appears to coincide with the most dorsal hemifield representation described by Kolster, labeled [2] within Figure 2 (Kolster et al., 2009) and may be functionally related to processes within the MT cluster but part of a different organizational cluster, perhaps also related to processes relevant to intrapersonal space occurring in the intraparietal sulcus (for review, see Vanduffel et al., 2014).

\section{MT}

The presence of position, velocity, direction, and disparitysensitive cells within MT are defining characteristics of the area (Zeki, 1974; Maunsell and Van Essen, 1983a, 1983b, Albright and Desimone, 1987; Rodman and Albright, 1987; DeAngelis and Newsome, 1999; DeAngelis and Uka, 2003) and its role in smooth pursuit movements was established by Newsome et al. (1985) and extended by Komatsu and Wurtz (1989) and Takemura et al. (2007). The same neural sensitivities and involvement in pursuit movements could provide the basis for the initiation of vergence movements as well by establishing a 3D representation of visual space through the signaling of speed, direction, and disparity information related to stimuli that pass through space (DeAngelis and Newsome, 2004; Krug et al., 2013). Figure $8 B$ shows clearly that all four movement types are well correlated with MR signal modulation within area MT. Together with the study by Bakola et al. (2007), which found saccade-related processing within MT, and the study by Baker et al. (2006), which found widespread saccade-related BOLD modulation with STS, including area MT, our results strengthen the role of area MT in the production of eye movements, both pursuit and stepped, in 3D space.

\section{V4t}

The myeloarchetecturally defined borders of V4t as defined by Desimone and Ungerleider (1986) are confined within the STS and its posterior lip and generally border the full length of MT. The two peaks of activation we observed with respect to saccade dominance (Fig. 7) appear to correspond to the subdivisions of V4t (V4tp and V4ta) illustrated by Van Essen et al. (2001) (Fig. 2). The dorsal node labeled V4t in Figure 7 coincides well with V4t within the frameworks of Felleman and Van Essen (1991), Paxinos et al. (2000), and V4ta in the Lewis and Van Essen, 2000a,2000b framework. However, a recent retinotopic study by Janssens et al. (2014) suggests that this region should be considered the anterior aspect of dorsal V4 (for parcellation scheme comparisons, see Fig. 9 in Janssens et al., 2014). The ventral node we label as V4t is somewhat less definite because the aforementioned parcellation frameworks show less congruence with respect to this area's identification. Depending on the framework referenced, this node could be included in V4t (Felleman and Van Essen, 1991; Lewis and Van Essen, 2000a,2000b), FST (Paxinos et al., 2000), or the dorsal occipitotemporal area (Janssens et al., 2014; Kolster et al., 2014). Given the evidence already supporting V4t's functional role in the production of saccades, both visually and memory guided (Bakola et al., 2007), and the direct projections it receives from saccade-related FEF (Stanton et al., 1995), we have used the V4t identification here until a more detailed and higher-resolution study can be conducted to resolve these discrepancies.

\section{Lower STS: FST, LST, PITd}

The robust and consistent modulation that we have demonstrated in areas located in the lower STS during eye movements is somewhat surprising given this area's complex and specific visual response properties and its association with object recognition and 3D visual processing (for review, see Orban, 2011). Area FST is known to possess properties related to the processing of $2 \mathrm{D}$ and 3D shape from motion (Sereno et al., 2002, 2005; Vanduffel et al., 2002) and Nelissen et al. (2006) provided further insight into the functional role of FST by positing that it contains directionally selective cells or opponent motion-selective neurons. Within the same study, Nelissen et al. described a new motion-sensitive area, LST, positioned anterior to FST within architectonic area IPa that also displayed 2D shape selectivity. Finally, area PITd, identified by Felleman and Van Essen (1991) and reinforced by Kolster et al. $(2009,2014)$ and located ventral to V4a spanning the bank and posterior gyrus of the STS, showed consistent modulation across eye movement types. Little is known about the functional properties of this region, but Janssen et al. (2000) reported neurons in this region that were selective for $2 \mathrm{D}$ and $3 \mathrm{D}$ shape as well. Further, given this region's connections with neurons within the caudal bank of the IPS that are selective for disparity-defined orientation, it is plausible that this region is a part of a network involved in the processing of 3D structure.

A different interpretation of the role that area PITd may play in visually guided eye movements comes from a recent study by Sereno et al. (2014). By analyzing eye-position-related neuronal modulation within the extremes of the dorsal-ventral stream dichotomy (i.e., lateral intraparietal area, LIP, and anterior inferotemporal cortex, AIT, respectively) the authors demonstrated a representation of visual space, not only where it might be expected within the dorsal stream (LIP), but also in the far ventral stream as well (AIT). These findings raise the possibility that other, intermediate areas within and along the STS (e.g., FST, LST) may also be involved in the representation of visual space, albeit with subtle differences and their output may be used for different purposes.

The scope of the present study was, by design, broad and did not address the question of the relative influences of visual-, motor-, visuomotor-, or feedback-related processing (with the exception of the VERGENCE TRACKING task). Given the specific and distinct functional properties that characterize the numerous areas within the STS, it is highly unlikely that the robust modulation we have documented is primarily due to the relatively simple visual stimuli used in the study. Therefore, further study is needed to determine: (1) to what degree the stimuli alone drive these areas and the level of dependence any stimulus-driven modulation has with respect to whether the stimulus is an eye movement target or a distractor, (2) how much of an area's mod- 
ulation is due to motor commands versus recurrent feedback from higher-order areas, and (3) to what degree aspects common to all tasks (e.g., eye position information, visual space map construction/updating) influence local BOLD signal modulation.

\section{References}

Akao T, Mustari MJ, Fukushima J, Kurkin S, Fukushima K (2005) Discharge characteristics of pursuit neurons in MST during vergence eye movements. J Neurophysiol 93:2415-2434. CrossRef Medline

Albright TD, Desimone R (1987) Local precision of visuotopic organization in the middle temporal area (MT) of the macaque. Exp Brain Res 65:582592. Medline

Baker JT, Patel GH, Corbetta M, Snyder LH (2006) Distribution of activity across the monkey cerebral cortical surface, thalamus and midbrain during rapid, visually guided saccades. Cereb Cortex 16:447-459. Medline

Bakola S, Gregoriou GG, Moschovakis AK, Raos V, Savaki HE (2007) Saccade-related information in the superior temporal motion complex: Quantitative functional mapping in the monkey. J Neurosci 27:22242229. CrossRef Medline

Breveglieri R, Hadjidimitrakis K, Bosco A, Sabatini SP, Galletti C, Fattori P (2012) Eye position in three-dimensional space: integration of version and vergence signals in medial posterior parietal cortex. J Neurosci 32: 159-169. CrossRef Medline

DeAngelis GC, Newsome WT (1999) Organization of disparity-selective neurons in macaque area MT. J Neurosci 19:1398-1415. Medline

DeAngelis GC, Newsome WT (2004) Perceptual "read-out" of conjoined direction and disparity maps in extrastriate area MT. PLoS Biol 2:E77. CrossRef Medline

DeAngelis GC, Uka T (2003) Coding of horizontal disparity and velocity by MT neurons in alert macaque. J Neurophysiol 89:1094-1111. Medline

Desimone R, Ungerleider LG (1986) Multiple visual areas in the caudal superior temporal sulcus of the macaque. J Comp Neurol 248:164-189. CrossRef Medline

Distler C, Hoffmann KP (2008) Private lines of cortical visual information to the nucleus of the optic tract and dorsolateral pontine nucleus. Prog Brain Res 171:363-368. CrossRef Medline

Duffy CJ, Wurtz RH (1991) Sensitivity of MST neurons to optic flow stimuli. I. A continuum of response selectivity to large-field stimuli. J Neurophysiol 65:1329-1345. Medline

Dürsteler MR, Wurtz RH (1988) Pursuit and optokinetic deficits following chemical lesions of cortical areas MT and MST. J Neurophysiol 60:940965. Medline

Eifuku S, Wurtz RH (1998) Response to motion in extrastriate area MSTl: center-surround interactions. J Neurophysiol 80:282-296. Medline

Eifuku S, Wurtz RH (1999) Response to motion in extrastriate area MSTl: disparity sensitivity. J Neurophysiol 82:2462-2475. Medline

Felleman DJ, Van Essen DC (1991) Distributed hierarchical processing in the primate cerebral cortex. Cereb Cortex 1:1-47. Medline

Fukushima J, Akao T, Takeichi N, Kurkin S, Kaneko CR, Fukushima K (2004) Pursuit-related neurons in the supplementary eye fields: discharge during pursuit and passive whole body rotation. J Neurophysiol 91:2809-2825. CrossRef Medline

Fukushima K, Yamanobe T, Shinmei Y, Fukushima J, Kurkin S, Peterson BW (2002) Coding of smooth eye movements in three-dimensional space by frontal cortex. Nature 419:157-162. CrossRef Medline

Gamlin PD (2002) Neural mechanisms for the control of vergence eye movements. Ann N Y Acad Sci 956:264-272. CrossRef Medline

Gamlin PD, Yoon K (2000) An area for vergence eye movements in primate frontal cortex. Nature 407:1003-1007. CrossRef Medline

Gamlin PD, Ward MK, Bolding MS, Grossmann JK, Twieg DB (2006) Developing functional magnetic resonance imaging techniques for alert macaque monkeys. Methods 38:210-220. CrossRef Medline

Hoffmann KP, Bremmer F, Distler C (2009) Visual response properties of neurons in cortical areas MT and MST projecting to the dorsolateral pontine nucleus or the nucleus of the optic tract in macaque monkeys. Eur J Neurosci 29:411-423. CrossRef Medline

Janssen P, Vogels R, Orban GA (2000) Selectivity for 3D shape that reveals distinct areas within macaque inferior temporal cortex. Science 288: 2054-2056. CrossRef Medline

Janssens T, Zhu Q, Popivanov ID, Vanduffel W (2014) Probabilistic and single-subject retinotopic maps reveal the topographic organization of face patches in the macaque cortex. J Neurosci 34:10156-10167. CrossRef Medline

Jenkinson M (2003) Fast, automated, N-dimensional phase-unwrapping algorithm. Magn Reson Med 49:193-197. CrossRef Medline

Jenkinson M, Bannister P, Brady M, Smith S (2002) Improved optimization for the robust and accurate linear registration and motion correction of brain images. Neuroimage 17:825-841. CrossRef Medline

Kolster H, Mandeville JB, Arsenault JT, Ekstrom LB, Wald LL, Vanduffel W (2009) Visual field map clusters in macaque extrastriate visual cortex. J Neurosci 29:7031-7039. CrossRef Medline

Kolster H, Janssens T, Orban GA, Vanduffel W (2014) The retinotopic organization of macaque occipitotemporal cortex anterior to V4 and caudoventral to middle temporal (MT) cluster. J Neurosci 34:10168-10191. CrossRef Medline

Komatsu H, Wurtz RH (1988a) Relation of cortical areas MT and MST to pursuit eye movements. I. Localization and visual properties of neurons. J Neurophysiol 60:580-603. Medline

Komatsu H, Wurtz RH (1988b) Relation of cortical areas MT and MST to pursuit eye movements. III. Interaction of full-field visual stimulation. J Neurophysiol 60:621-644. Medline

Komatsu H, Wurtz RH (1989) Modulation of pursuit eye movements by stimulation of cortical areas MT and MST. J Neurophysiol 62:31-47. Medline

Krauzlis RJ (2004) Recasting the smooth pursuit eye movement system. J Neurophysiol 91:591-603. Medline

Krug K, Cicmil N, Parker AJ, Cumming BG (2013) A causal role for V5/MT neurons coding motion-disparity conjunctions in resolving perceptual ambiguity. Curr Biol 23:1454-1459. Medline

Lewis JW, Van Essen DC (2000a) Mapping of architectonic subdivisions in the macaque monkey, with emphasis on parieto-occipital cortex. J Comp Neurol 428:79-111. CrossRef Medline

Lewis JW, Van Essen DC (2000b) Corticocortical connections of visual, sensorimotor, and multimodal processing areas in the parietal lobe of the macaque monkey. J Comp Neurol 428:112-137. CrossRef Medline

Lynch JC, Tian JR (2006) Cortico-cortical networks and cortico-subcortical loops for the higher control of eye movements. Prog Brain Res 151:461501. CrossRef Medline

Maunsell JH, Van Essen DC (1983a) Functional properties of neurons in middle temporal visual area of the macaque monkey. I. Selectivity for stimulus direction, speed and orientation. J Neurophysiol 49:1127-1147. Medline

Maunsell JH, Van Essen DC (1983b) Functional properties of neurons in middle temporal visual area of the macaque monkey. II. Binocular interactions and sensitivity to binocular disparity. J Neurophysiol 49:11481167. Medline

Miyasaka N, Takahashi K, Hetherington HP (2006) Fully automated shim mapping method for spectroscopic imaging of the mouse brain at $9.4 \mathrm{~T}$. Magn Reson Med 55:198-202. CrossRef Medline

Nelissen K, Vanduffel W, Orban GA (2006) Charting the lower superior temporal region, a new motion sensitive region in the monkey superior temporal sulcus. J Neurosci 26:5929-5947. CrossRef Medline

Newsome WT, Wurtz RH, Dürsteler MR, Mikami A (1985) Deficits in visual motion processing following ibotenic acid lesions of the middle temporal visual area of the macaque monkey. J Neurosci 5:825-840. Medline

Newsome WT, Wurtz RH, Komatsu H (1988) Relation of cortical areas MT and MST to pursuit eye movements. II. Differentiation of retinal from extraretinal inputs. J Neurophysiol 60:604-620. Medline

Orban GA (2011) The extraction of 3D shape in the visual system of human and nonhuman primates. Annu Rev Neurosci 34:361-388. CrossRef Medline

Paxinos G, Huang XF, Toga AW (2000) The rhesus monkey brain in stereotaxic coordinates. Orlando, FL: Academic.

Rodman HR, Albright TD (1987) Coding of visual stimulus velocity in area MT of the macaque. Vision Res 27:2035-2048. Medline

Sereno AB, Sereno ME, Lehky SR (2014) Recovering stimulus locations using populations of eye-position modulated neurons in dorsal and ventral visual streams of non-human primates. Front Integr Neurosci 8:28. Medline

Sereno ME, Trinath T, Augath M, Logothetis NK (2002) Three-dimensional shape representation in monkey cortex. Neuron 33:635-652. CrossRef Medline 
Sereno ME, Augath, Logothetis NK (2005) Differences in processing of 2Dshape from multiple cues in monkey cortex revealed by fMRI. Soc Neurosci Abstr 31:362.9.

Smith SM (2002) Fast robust automated brain extraction. Hum Brain Mapp 17:143-155. CrossRef Medline

Smith SM, Jenkinson M, Woolrich MW, Beckmann CF, Behrens TEJ, Johansen-Berg H, Bannister PR, De Luca M, Drobnjak I, Flitney DE, Niazy RK, Saunders J, Vickers J, Zhang Y, De Stefano N, Brady JM, Matthews PM (2004) Advances in functional and structural MR image analysis and implementation as FSL. Neuroimage 23:S208-S219. CrossRef Medline

Stanton GB, Bruce CJ, Goldberg ME (1995) Topography of projections to the posterior cortical areas from the macaque frontal eye fields. J Comp Neurol 353:291-305. CrossRef Medline

Stanton GB, Friedman HR, Dias EC, Bruce CJ (2005) Cortical afferents to the smooth-pursuit region of the macaque monkey's frontal eye field. Exp Brain Res 165:179-192. CrossRef Medline

Takemura A, Murata Y, Kawano K, Miles FA (2007) Deficits in shortlatency tracking eye movements after chemical lesions in monkey cortical areas MT and MST. J Neurosci 27:529-541. CrossRef Medline

Tanaka K, Sugita Y, Moriya M, Saito H (1993) Analysis of object motion in the ventral part of the medial superior temporal area of the macaque. J Neurophysiol 69:128-142. Medline

Van Essen DC (2002) Windows on the brain: the emerging role of atlases and databases in neuroscience. Curr Opin Neurobiol 12:574-579. CrossRef Medline

Van Essen DC, Lewis JW, Drury HA, Hadjikhani N, Tootell RB, Bakircioglu M, Miller MI (2001) Mapping visual cortex in monkeys and humans using surface-based atlases. Vision Res 41:1359-1378. CrossRef Medline

Van Essen DC, Drury HA, Dickson J, Harwell J, Hanlon D, Anderson CH (2001) An integrated software suite for surface-based analysis of cerebral cortex. J Am Med Inform Assoc 8:443-459. CrossRef Medline

Van Horn MR, Waitzman DM, Cullen KE (2013) Vergence neurons identified in the rostral superior colliculus code smooth eye movements in 3D space. J Neurosci 33:7274-7284. CrossRef Medline

Vanduffel W, Fize D, Peuskens H, Denys K, Sunaert S, Todd JT, Orban GA (2002) Extracting 3D from motion: differences in human and monkey intraparietal cortex. Science 298:413-415. CrossRef Medline

Vanduffel W, Zhu Q, Orban GA (2014) Monkey cortex through fMRI glasses. Neuron 83:533-550. CrossRef Medline

Woolrich MW, Ripley BD, Brady M, Smith SM (2001) Temporal autocorrelation in univariate linear modeling of FMRI data. Neuroimage 14: 1370-1386. CrossRef Medline

Zeki SM (1974) Functional organization of a visual area in the posterior bank of the superior temporal sulcus of the rhesus monkey. J Physiol 236:549-573. CrossRef Medline

Zemel RS, Sejnowski TJ (1998) A model for encoding multiple object motions and self-motion in area MST of primate visual cortex. J Neurosci 18:531-547. Medline 